\title{
Ultrafast Reversal of the Ferroelectric Polarization
}

\author{
R. Mankowsky, ${ }^{1}$ A. von Hoegen, ${ }^{1}$ M. Först, ${ }^{1}$ and A. Cavalleri ${ }^{1,2}$ \\ ${ }^{1}$ Max Planck Institute for the Structure and Dynamics of Matter, 22761 Hamburg, Germany \\ ${ }^{2}$ Department of Physics, University of Oxford, Clarendon Laboratory, Oxford OX1 3PU, United Kingdom
}

(Received 21 January 2017; published 10 May 2017)

\begin{abstract}
We report on the demonstration of ultrafast optical reversal of the ferroelectric polarization in $\mathrm{LiNbO}_{3}$. Rather than driving the ferroelectric mode directly, we couple to it indirectly by resonant excitation of an auxiliary high-frequency phonon mode with femtosecond midinfrared pulses. Because of strong anharmonic coupling between these modes, the atoms are directionally displaced along the ferroelectric mode and the polarization is transiently reversed, as revealed by time-resolved, phase-sensitive, secondharmonic generation. This reversal can be induced in both directions, a key prerequisite for practical applications.
\end{abstract}

DOI: 10.1103/PhysRevLett.118.197601

The ferroelectric polarization is typically controlled with static or pulsed electric fields. Switching is, in this case, an incoherent process, with speed limited to hundreds of picoseconds by the nucleation and growth of oppositely polarized domains [1-3]. To overcome these limitations, attempts have been made to drive the ferroelectric mode coherently with light pulses. These strategies, which have been based either on impulsive Raman scattering [4-7] or direct excitation of the ferroelectric mode [8-10] using $\mathrm{THz}$ radiation, have not yet been completely successful.

Recent theoretical work [11] has analyzed an alternative route to manipulate the ferroelectric polarization on ultrafast time scales. It has been proposed that a coherent displacement of the ferroelectric mode could be achieved indirectly, by exciting a second, anharmonically coupled vibrational mode at higher frequency. The underlying mechanism is captured by the following minimal model, which is illustrated in Fig. 1. Consider a double well energy potential along the ferroelectric mode coordinate $Q_{P}$ as shown in Fig. 1(b) (dashed black line)

$$
V_{P}\left(Q_{P}\right)=-\frac{1}{4} \omega_{P}^{2} Q_{P}^{2}+\frac{1}{4} c_{p} Q_{P}^{4} .
$$

Here, $\omega_{p}$ is the frequency of the ferroelectric mode for a fixed temperature $T$ below the Curie temperature $T_{C}$. Take then a second mode $Q_{\mathrm{IR}}$ with frequency $\omega_{\mathrm{IR}}$, described by the harmonic potential $V_{\mathrm{IR}}\left(Q_{\mathrm{IR}}\right)=\frac{1}{2} \omega_{\mathrm{IR}}^{2} Q_{\mathrm{IR}}^{2}$ shown in Fig. 1(a), which is anharmonically coupled to the ferroelectric mode with a quadratic-linear dependence of the interaction energy $a Q_{\mathrm{IR}}^{2} Q_{P}$. The total lattice potential can then be written as

$$
V\left(Q_{P}, Q_{\mathrm{IR}}\right)=V_{P}\left(Q_{P}\right)+V_{\mathrm{IR}}\left(Q_{\mathrm{IR}}\right)-a Q_{\mathrm{IR}}^{2} Q_{P} .
$$

At equilibrium, $Q_{P}$ takes the value of one of the two minima of the double well potential $V_{P}\left(Q_{P}\right)$ [dashed black line in Fig. 1(b)]. For finite displacements $Q_{\mathrm{IR}}$, this minimum is first displaced and then destabilized as $Q_{\mathrm{IR}}$ exceeds a threshold value [colored lines in Fig. 1(b)]. Importantly, the direction of this displacement is always pointed toward the opposite potential well and independent on the sign of $Q_{\mathrm{IR}}$ [11].

The corresponding dynamics of the two modes for resonant periodic driving of $Q_{\mathrm{IR}}$ by a midinfrared light pulse is obtained by solving the equations of motion

$$
\begin{aligned}
& \ddot{Q}_{\mathrm{IR}}+\gamma_{\mathrm{IR}} \dot{Q}_{\mathrm{IR}}+\omega_{\mathrm{IR}}^{2} Q_{\mathrm{IR}}=2 a Q_{P} Q_{\mathrm{IR}}+f(t), \\
& \ddot{Q}_{P}+\gamma_{P} \dot{Q}_{P}-1 / 2 \omega_{P}^{2} Q_{P}+c_{P} Q_{P}^{3}=a Q_{\mathrm{IR}}^{2} .
\end{aligned}
$$

Here, $f(t)=A \sin \left(\omega_{\mathrm{IR}} t\right) \exp \left(-4 \ln 2 t^{2} / T^{2}\right)$ is the driving pulse with Gaussian envelope of duration $T$ and frequency $\omega_{\mathrm{IR}}$. As displayed in Fig. 1(c), the driven mode $Q_{\mathrm{IR}}$ experiences oscillations about its equilibrium position, whereas the ferroelectric mode $Q_{P}$ is subject to a unidirectional force $\partial V / \partial Q_{P}=a Q_{\mathrm{IR}}^{2}[12,13]$.

Two regimes are found for the response of the ferroelectric mode, shown in Fig. 1(d). For small oscillation amplitudes in $Q_{\mathrm{IR}}$, the energy minimum of the double well potential undergoes a prompt displacement, launching oscillations of $Q_{P}$ around a new position. In this case, the ferroelectric polarization is reduced but retains at all times the same sign of the equilibrium state. For oscillation amplitudes of $Q_{\mathrm{IR}}$ in excess of a threshold, the model predicts that the original energy minimum is destabilized and $Q_{P}$ is moved to the opposite potential well, switching the polarization. A similar conversion of driven oscillatory motions into directional displacements has been previously demonstrated in a number of nonferroelectric materials [14-16].

Here, we seek to validate these ideas experimentally for the case of $\mathrm{LiNbO}_{3}$, a rhombohedrally distorted perovskite ferroelectric with Curie temperature $T_{C}=1210^{\circ} \mathrm{C}$. The structure of ferroelectric $\mathrm{LiNbO}_{3}$ is displayed in Fig. 1(e). Below $\mathrm{T}_{C}$, the $\mathrm{Li}$ and $\mathrm{Nb}$ sublattices move against the oxygen octahedra, inducing a stable electrical polarization 


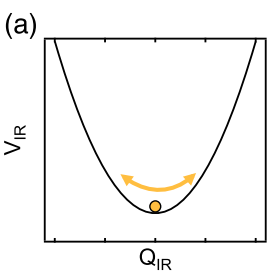

(b)

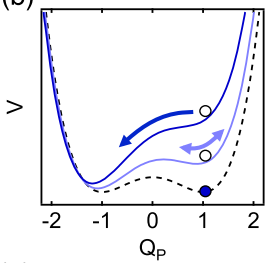

(e)

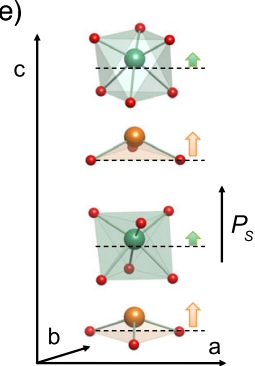

(c)

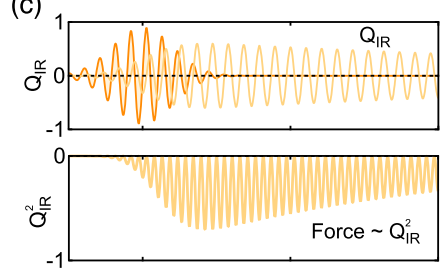

(d)
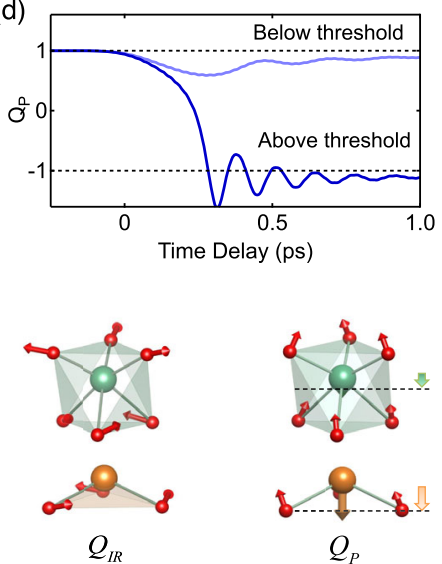

○i $\bigcirc \mathrm{Nb} \bullet \mathrm{O}$

FIG. 1. (a) Harmonic energy potential $V_{\mathrm{IR}}\left(Q_{\mathrm{IR}}\right)$ of the resonantly excited high frequency mode $Q_{\mathrm{IR}}$. (b) Double well potential $V\left(Q_{P}, Q_{\mathrm{IR}}\right)$ along the ferroelectric mode $Q_{P}$. For finite amplitude of $Q_{\mathrm{IR}}$, the energy minimum of the equilibrium potential [ $V_{P}\left(Q_{P}\right)$, dashed line] is first displaced and then destabilized as the amplitude of $Q_{\mathrm{IR}}$ exceeds a threshold (colored lines). (c),(d) Solution to the corresponding coupled equations of motion (see text). Following excitation of $Q_{\mathrm{IR}}$ with midinfrared pulses (orange), a force $F \propto Q_{\mathrm{IR}}^{2}$ displaces the ferroelectric mode $Q_{P}$ toward lower values. For above-threshold excitation of $Q_{\mathrm{IR}}$, the polarization reverses permanently. (e) Crystal structure of ferroelectric $\mathrm{LiNbO}_{3}$. Drawn are the hexagonal crystal axes. The $\mathrm{Li}$ an $\mathrm{Nb}$ sublattices are shifted against the oxygen octahedra (orange and green arrows), inducing a ferroelectric polarization $P_{S}$ along the $c$ axis. Further shown are schematics of the motions associated with the phonon modes $Q_{\mathrm{IR}}$ and $Q_{P}$. The ferroelectric mode $Q_{P}$ involves $c$-axis motions of the $\mathrm{Nb}$ and Li sublattices against the oxygen octahedra (orange and green arrows) and modulates the ferroelectric polarization.

along the rhombohedral $(111)_{r}$ direction that corresponds to the hexagonal $c$ axis. This structural transition involves atomic motions along three normal modes, one of which causes the ferroelectric polarization. Density functional theory calculations reveal strong anharmonic coupling of this $7.5-\mathrm{THz}$ ferroelectric mode $Q_{P}$ to a high-frequency $19 \mathrm{THz}$ mode $Q_{\mathrm{IR}}$, just as described in the model above [11]. The atomic motions associated with these phonon modes are shown in Fig. 1(e).

We resonantly excited the $19 \mathrm{THz} Q_{\mathrm{IR}}$ mode in a $5-\mathrm{mm}$ thick $x$-cut single-domain $\mathrm{LiNbO}_{3}$ crystal using $150 \mathrm{fs}$ midinfrared pulses. Figure 2(a) shows the electric field and Fourier transform of the $19 \mathrm{THz}$ pump pulses, which were obtained by optical parametric amplification and difference frequency generation. The penetration depth of these

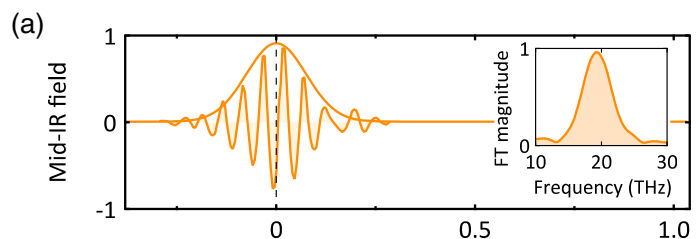

(b)

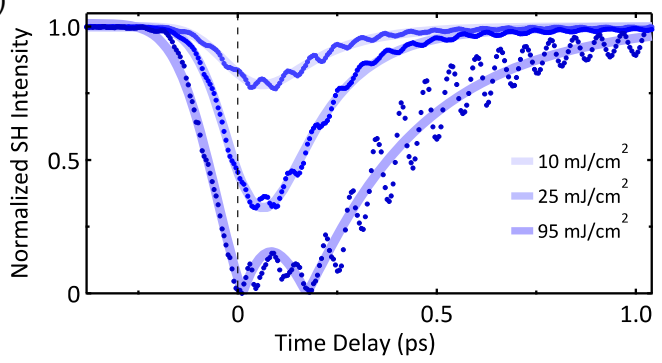

FIG. 2. (a) Carrier envelope phase stable $19 \mathrm{THz}$ midinfrared pulses were used to excite the high frequency mode $Q_{\mathrm{IR}}$ shown in Fig. 1(e). The inset shows the Fourier transformation of the pulse. (b) Time-resolved second-harmonic intensity, normalized to its value before excitation. Following excitation, the second-harmonic intensity reduces before relaxing back. This drop increases with pump fluence. For all fluences above $60 \mathrm{~mJ} / \mathrm{cm}^{2}$, the secondharmonic signal vanishes, followed by a transient recovery before relaxing back to its equilibrium value.

midinfrared pulses was $3.2 \mu \mathrm{m}$. The ferroelectric polarization dynamics were measured by recording the transmitted second-harmonic ( $\mathrm{SH}$ ) signal generated by a $35 \mathrm{fs}, 800-\mathrm{nm}$ wavelength probe pulse, which was delayed in time with respect to the excitation. As the detected SH signal was generated in the first $1.3 \mu \mathrm{m}$ of the crystal, the probed volume was homogeneously excited (see Supplemental Material, Sec. I for details [17]).

Time-resolved measurements of the SH intensity $I_{\mathrm{SH}} \propto$ $\left|\varepsilon_{0} \chi^{(2)} E_{\text {Probe }}^{2}(t)\right|^{2}$ yielded the magnitude of the time dependent second order susceptibility $\chi^{(2)}$, which is directly proportional to the polarization $P_{S}$ [24]. Higher order contributions near the second-harmonic frequency $2 \omega_{\text {probe }}$, most notably those descending from cubic $\chi^{(3)}$ nonlinearities at frequencies $2 \omega_{\text {probe }}+\omega_{\text {MIR }}$ and $2 \omega_{\text {probe }}-\omega_{\text {MIR }}$, were filtered spatially as described in the Supplemental Material, Sec. II [17].

The time-resolved second-harmonic intensity is plotted in Fig. 2(b), normalized to its equilibrium value. For excitation at fluences $F<50 \mathrm{~mJ} / \mathrm{cm}^{2}$, we observed a reduction in SH intensity to a finite value within approximately $200 \mathrm{fs}$. As predicted by the model presented above, we observed a rapid exponential recovery and coherent oscillations at $16 \mathrm{THz}$, which we attribute to a phonon-polariton mode $[25,26]$, associated with the driven $Q_{\mathrm{IR}}$ phonon.

For fluences above a threshold value of $60 \mathrm{~mJ} / \mathrm{cm}^{2}$ and up to the maximum fluence possible in our setup $\left(95 \mathrm{~mJ} / \mathrm{cm}^{2}\right)$, the second-harmonic intensity was observed to vanish completely, recover to a finite value, and then vanish again, before relaxing back to the equilibrium state. 
(a)

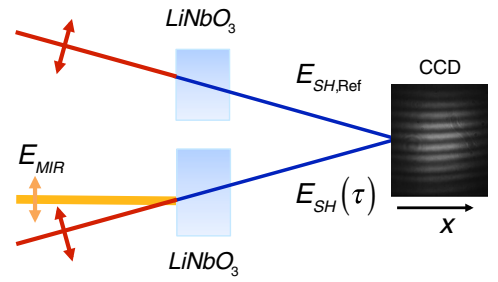

(b)

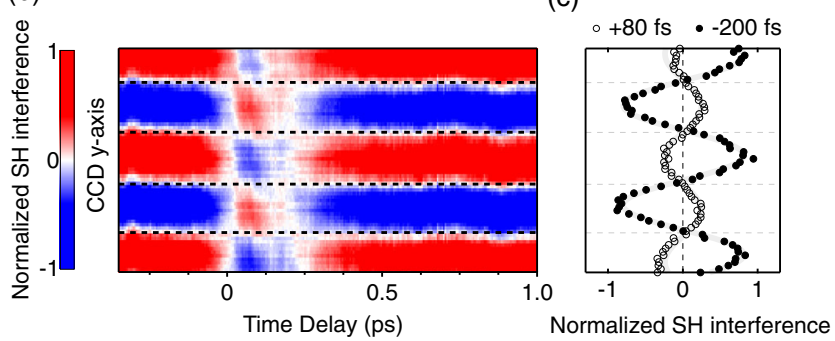

FIG. 3. (a) Experimental geometry for phase-sensitive measurements of the second harmonic. The time dependent secondharmonic signal from the excited $\mathrm{LiNbO}_{3}$ crystal was interfered with a reference second-harmonic field from an unexcited sample and measured with a CCD camera. The resulting interference pattern consisted of fringes on top of a Gaussian background, which was subtracted. (b) Time-resolved measurement of the interference fringes, integrated along the $x$ axis of the camera and normalized. The data show a transient phase change by $180^{\circ}$ (sign reversal) between 0 and 200 fs. (c) Normalized interference fringes at $-200 \mathrm{fs}$ and in the reversed polarization state at $+80 \mathrm{fs}$.

To derive whether the ferroelectric polarization was reversed during this dynamics, we measured the timedependent phase of the SH electric field by interfering it with a reference $\mathrm{SH}$ pulse, generated in a nonexcited crystal as sketched in Fig. 3(a). The resulting pattern, which consisted of interference fringes on top of a Gaussian background, was detected with a charge coupled device (CCD) camera. For further analysis, this background was subtracted (see Supplemental Material, Sec. II [17]). Changes in the phase of the second-harmonic signal generated in the driven $\mathrm{LiNbO}_{3}$ crystal appeared as changes in the spatial position of these fringes on the camera.

Figure 3(b) displays the time dependent interference signal integrated along the fringe direction for the maximum pump fluence of $95 \mathrm{~mJ} / \mathrm{cm}^{2}$. After excitation, the $\mathrm{SH}$ intensity first reduced with a constant phase. As the intensity reached zero at $0 \mathrm{fs}$ time delay, the phase of the second-harmonic field flipped by $180^{\circ}$, as revealed by a sudden sign change of the interference fringes. This sign change is clearly visible in Fig. 3(c), in which the interference fringes are shown for a negative delay at $-200 \mathrm{fs}$ and in the reversed state at $+80 \mathrm{fs}$. The phase then remained constant until the SH intensity vanished again at 200 fs time delay, when the phase switched back to the initial value. Thus, for time delays between 0 and $200 \mathrm{fs}$, the polarization was transiently reversed.

A deeper understanding of the reversal process was obtained by analyzing the time dependent changes in $\mathrm{SH}$
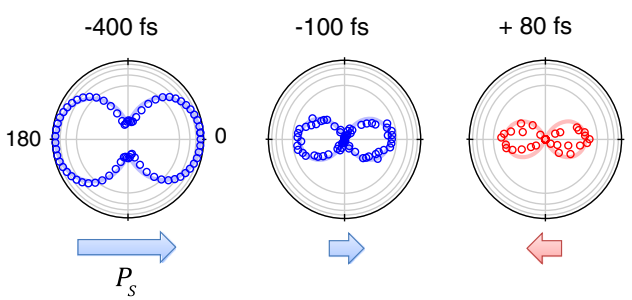

$+400 \mathrm{fs}$

FIG. 4. Probe pulse polarization dependence of the secondharmonic signal at four time delays after excitation. The angles $0^{\circ}$ and $90^{\circ}$ denote a polarization of the $800 \mathrm{~nm}$ pulses along and perpendicular to the spontaneous polarization. The arrows indicate the time dependent amplitude and sign of the ferroelectric polarization for each time delay, determined from the measurements of Fig. 3. The solid lines are fits to the data using the $\chi^{(2)}$ tensor of $\mathrm{LiNbO}_{3}$.

intensity for different probe polarizations. As shown in Fig. 4, the polarization dependence of the second harmonic retained the same symmetry and shape of that observed at equilibrium for all time delays. Hence, the dynamical reversal occurs only along the hexagonal $c$ axis with no rotations in the plane, consistent with atomic displacements along the ferroelectric mode $Q_{P}$ as described by the model above.

We further tested a second key element of the model, according to which the force acting on $Q_{P}$ depends on the initial equilibrium polarization state. For a polarization pointing "up," the force acts toward the "down" state, and vice versa [11]. Polarization reversal should thus be observed starting from both equilibrium states without changing the pump pulse characteristics. In Fig. 5 we present the normalized amplitude (a) and phase (b) of the time dependent ferroelectric polarization measured from both initial polarization states, as extracted from the secondharmonic intensity and phase according to $P_{S}(\tau) / P_{S, 0}=$ $\sqrt{I_{\mathrm{SH}} / I_{\mathrm{SH}, 0}} e^{i \varphi(\tau)}+$ c.c.. As predicted, the sign of the polarization could be transiently reversed, with a similar dynamical evolution in the two cases.

According to the model, the ferroelectric polarization reversal should further exhibit a threshold. Figure 6(a) shows a fluence dependence of the polarization at the peak of the time-resolved signal, normalized to its equilibrium value $\left[P_{S}(\tau) / P_{S, 0}\right]_{\text {peak }}$, for different excitation frequencies. Indeed, we find that for excitation pulses resonant with the high-frequency $Q_{\mathrm{IR}}$ mode transverse optical frequency $\omega_{I R, \mathrm{TO}}$ of $19 \mathrm{THz}$, the polarization reverses only for fluences that exceed $60 \mathrm{~mJ} / \mathrm{cm}^{2}$.

We also find a substantial increase in threshold fluence for pump pulses detuned from the resonance condition. This effect is clearly captured in Fig. 6(b), where we plot the slopes of linear fits to the fluence dependence of $\left[P_{S}(\tau) / P_{S, 0}\right]_{\text {peak }}$, as shown in Fig. 6(a), for different pump pulse frequencies. This susceptibility closely follows the extinction coefficient of $\mathrm{LiNbO}_{3}$, and peaks at $\omega_{\text {IR.TO }}$, which is again in agreement with the above model of 


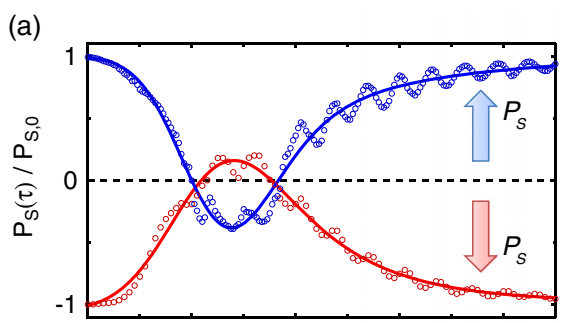

(b)

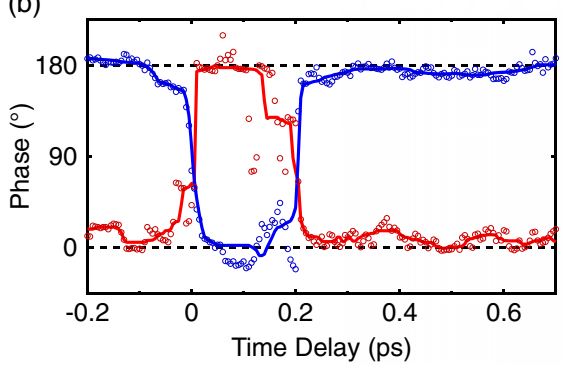

FIG. 5. (a) Time-resolved normalized ferroelectric polarization $P_{S}(\tau) / P_{S, 0}$ following excitation of $\mathrm{LiNbO}_{3}$ with two opposite initial polarization states, as determined from the measurement of the SH intensity and the phase of the SH field. (b) Phase of the SH field derived from a sine fit to the interference pattern (see text).

anharmonic coupling between the driven mode $Q_{\mathrm{IR}}$ and the ferroelectric mode $Q_{P}$.

Hence, the reversal without rotation, the bidirectional nature of the effect, the existence of a switching threshold and the resonance with the frequency of the infrared mode, all indicate that the essence of this phenomenon is well understood in terms of anharmonic phonon coupling and directional displacement of the ferroelectric mode.

However, some issues require further investigation. The reversed polarization was found to be only $40 \%$ of its equilibrium value, which might be due to the limited fluence of $95 \mathrm{~mJ} / \mathrm{cm}^{2}$ available for $19 \mathrm{THz}$ pump pulses with our optical setup. This effect is not predicted by the minimal model and may be a sign of spatially inhomogeneous reversal. We envisage new experiments that make use of ultrafast diffuse $\mathrm{x}$-ray scattering with free electron lasers to quantify the possible inhomogeneity [27].

A second issue concerns the rapid return of the polarization to the initial state. In the model, switching to a stable state is expected when the threshold is exceeded. One explanation for this discrepancy may be that only coupling to the ferroelectric mode $Q_{P}$ is considered in our model. As described above, the equilibrium paraelectric-ferroelectric transition involves motion along three phonon modes. The crystal structure thus might have to relax along these two additional modes in order to permanently stabilize the reversed state. As the transient reversed state has a short lifetime, this relaxation might not occur here. Thus, permanent polarization reversal on femtosecond time scales may require selective excitation of additional vibrational modes with separate pulses to facilitate the relaxation. In the Supplemental Material, Sec. III we elaborate on this (a)

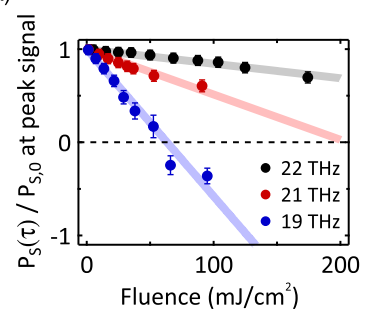

(b)

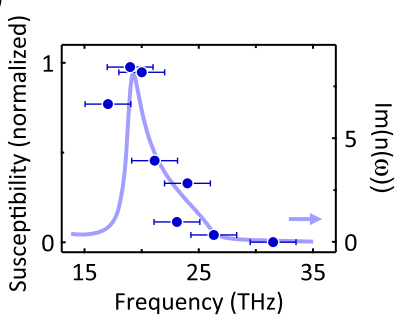

FIG. 6. (a) Normalized polarization at the peak of the time dependent signal $\left[P_{S}(\tau) / P_{S, 0}\right]_{\text {peak }}$ as function of pump fluence. The three curves correspond to excitation with pulses at different frequencies. The polarization reverses for excitation above $60 \mathrm{~mJ} / \mathrm{cm}^{2}$ with $19-\mathrm{THz}$ pulses, resonant with the $Q_{\mathrm{IR}}$ mode transverse optical frequency $\omega_{\mathrm{IR}, \mathrm{TO}}$. (b) Pump pulse frequency dependence of the susceptibility to the excitation (blue dots, left axis), defined as the slope of linear fits to the fluence dependence of $\left[P_{S}(\tau) / P_{S, 0}\right]_{\text {peak }}$, shown in Fig. 6(a). The solid line shows the extinction coefficient of $\mathrm{LiNbO}_{3}$ for comparison (right axis).

possibility, considering the contribution of more anharmonic terms to the lattice potential [17].

The rapid return to the initial polarization may also be explained by the formation of uncompensated charges after polarization reversal of only a fraction of the material. The use of thin films or other types of fabricated structures that isolate the reversed volume may improve the lifetime or clarify these points. As similar anharmonic coupling terms have been calculated for other perovskite transition-metal ferroelectrics [11] such as $\mathrm{PbTiO}_{3}$ or $\mathrm{BaTiO}_{3}$, the same technique could also be applied to materials with lower coercive fields.

Despite these limitations, which at the present stage make it impossible to build a nonvolatile ultrafast memory unit, immediate applications for the transient polarization reversal demonstrated here can already be envisaged. For example, the ability to reverse the polarization on femtosecond time scales may be used to control complex functionalities dynamically in materials that exhibit more than one coupled ferroic order [28-30] or to transfer charges at ultrafast speeds across heterointerfaces [31,32].

We thank Michael Fechner, Roberto Merlin, and Alaska Subedi for valuable discussions. The research leading to these results received funding from the European Research Council under the European Union's Seventh Framework Programme (FP7/2007-2013)/ERC Grant Agreement No. 319286 (QMAC). This work has been supported by the excellence cluster "The Hamburg Centre for Ultrafast Imaging - Structure, Dynamics and Control of Matter at the Atomic Scale" of the Deutsche Forschungsgemeinschaft.

[1] R. Landauer, Electrostatic considerations in $\mathrm{BaTiO}_{3}$ domain formation during polarization reversal, J. Appl. Phys. 28, 227 (1957). 
[2] J. Li, B. Nagaraj, H. Liang, W. Cao, Chi. H. Lee, and R. Ramesh, Ultrafast polarization switching in thin-film ferroelectrics, Appl. Phys. Lett. 84, 1174 (2004).

[3] K. Fujimoto and Y. Cho, Nanosecond switching of nanoscale ferroelectric domains in congruent single-crystal $\mathrm{LiTaO}_{3}$ using scanning nonlinear dielectric microscopy, Jpn. J. Appl. Phys. 43, 2818 (2004).

[4] S. Fahy and R. Merlin, Reversal of Ferroelectric Domains by Ultrashort Optical Pulses, Phys. Rev. Lett. 73, 1122 (1994).

[5] A. Cavalleri, S. Wall, C. Simpson, E. Statz, D. W. Ward, K. A. Nelson, M. Rini, and R. W. Schoenlein, Tracking the motion of charges in a terahertz light field by femtosecond x-ray diffraction, Nature (London) 442, 664 (2006).

[6] J. B. Ciaran and K. A. Nelson, Direct time-resolved measurement of anharmonic lattice vibrations in ferroelectric crystals, J. Chem. Phys. 107, 9691 (1997).

[7] K. Istomin, V. Kotaidis, A. Plech, and Q. Kong, Dynamics of the laser-induced ferroelectric excitation in $\mathrm{BaTiO}_{3}$ studied by x-ray diffraction, Appl. Phys. Lett. 90, 022905 (2007).

[8] T. Qi, Y.-H. Shin, K.-L. Yeh, K. A. Nelson, and A. M. Rappe, Collective Coherent Control: Synchronization of Polarization in Ferroelectric $\mathrm{PbTiO}_{3}$ by Shaped THz Fields, Phys. Rev. Lett. 102, 247603 (2009).

[9] S. Grübel, J. A. Johnson, P. Beaud, C. Dornes, A. Ferrer, V. Haborets, L. Huber, T. Huber, A. Kohutych, T. Kubacka, M. Kubli, S. O. Mariager, J. Rittmann, J. I. Saari, Y. Vysochanskii, G. Ingold, and S. L. Johnson, Ultrafast X-ray diffraction of a ferroelectric soft mode driven by broadband terahertz pulses, arXiv:1602.05435.

[10] F. Chen et al., Ultrafast terahertz-field-driven ionic response in ferroelectric $\mathrm{BaTiO}_{3}$, Phys. Rev. B 94, 180104(R) (2016).

[11] A. Subedi, Proposal for ultrafast switching of ferroelectrics using midinfrared pulses, Phys. Rev. B 92, 214303 (2015).

[12] A. Subedi, A. Cavalleri, and A. Georges, Theory of nonlinear phononics for coherent light control of solids, Phys. Rev. B 89, 220301 (2014).

[13] R. Mankowsky, M. Först, and A. Cavalleri, Nonequilibrium control of complex solids by nonlinear phononics, Rep. Prog. Phys. 79, 064503 (2016).

[14] M. Först, C. Manzoni, S. Kaiser, Y. Tomioka, Y. Tokura, R. Merlin, and A. Cavalleri, Nonlinear phononics as an ultrafast route to lattice control, Nat. Phys. 7, 854 (2011).

[15] M. Först, R. Mankowsky, H. Bromberger, D. M. Fritz, H. Lemke, D. Zhu, M. Chollet, Y. Tomioka, Y. Tokura, R. Merlin, J. P. Hill, S. L. Johnson, and A. Cavalleri, Displacive lattice excitation through nonlinear phononics viewed by femtosecond x-ray diffraction, Solid State Commun. 169, 24 (2013).

[16] R. Mankowsky, A. Subedi, M. Först, S. O. Mariager, M. Chollet, H. Lemke, J. Robinson, J. Glownia, M. Minitti, A. Frano, M. Fechner, N. Spaldin, T. Loew, M. Le Tacon, B. Keimer, A. Georges, and A. Cavalleri, Nonlinear lattice dynamics as a basis for enhanced superconductivity in $\mathrm{YBa}_{2} \mathrm{Cu}_{3} \mathrm{O}_{6.5}$, Nature (London) 516, 71 (2014).

[17] See Supplemental Material at http://link.aps.org/ supplemental/10.1103/PhysRevLett.118.197601, which includes Refs. [18-23], for details on the measurement technique, the experimental setup, and for an advanced model of the lattice anharmonicities.

[18] M. Mlejnek, E. M. Wright, J. V. Moloney, and N. Bloembergen, Second Harmonic Generation of Femtosecond Pulses at the Boundary of a Nonlinear Dielectric, Phys. Rev. Lett. 83, 2934 (1999).

[19] V. Roppo, M. Centini, C. Sibilia, M. Bertolotti, D. de Ceglia, M. Scalora, N. Akozbek, M. J. Bloemer, J. W. Haus, O. G. Kosareva, and V. P. Kandidov, Role of phase matching in pulsed second-harmonic generation: Walk-off and phase-locked twin pulses in negative-index media, Phys. Rev. A 76, 033829 (2007).

[20] N. Bloembergen and P. S. Pershan, Light Waves at the Boundary of Nonlinear Media, Phys. Rev. 128, 606 (1962).

[21] P. Schoen, Phase and Polarization Pulse Shaping for Nonlinear Microscopy (Université Paul Cézanne-AixMarseille III, 2010).

[22] E. Fazio, F. Pettazzi, M. Centini, M. Chauvet, A. Belardini, M. Alonzo, C. Sibilia, M. Bertolotti, and M. Scalora, Complete spatial and temporal locking in phase- mismatched second-harmonic generation, Opt. Express 17, 3141 (2009).

[23] SNLO nonlinear optics code available from A. V. Smith, AS-Photonics, Albuquerque, NM.

[24] R. C. Miller and A. Savage, Temperature dependence of the optical properties of ferroelectric $\mathrm{LiNbO}_{3}$ and $\mathrm{LiTaO}_{3}$, Appl. Phys. Lett. 9, 169 (1966).

[25] T. F. Crimmins, N. S. Stoyanov, and K. A. Nelson, Heterodyned impulsive stimulated Raman scattering of phononpolaritons in $\mathrm{LiTaO}_{3}$ and $\mathrm{LiNbO}_{3}$, J. Chem. Phys. 117, 2882 (2002).

[26] T. P. Dougherty, G. P. Wiederrecht, and K. A. Nelson, Impulsive stimulated Raman scattering experiments in the polariton regime, J. Opt. Soc. Am. B 9, 2179 (1992).

[27] A. Barty, S. Boutet, M. J. Bogan, S. Hau-Riege, S. Marchesini, K. Sokolowski-Tinten, N. Stojanovic, R. Tobey, H. Ehrke, A. Cavalleri, St. Düsterer, M. Frank, S. Bajt, B. W. Woods, M. M. Seibert, J. Hajdu, R. Treusch, and H. N. Chapman, Ultrafast single-shot diffraction imaging of nanoscale dynamics, Nat. Photonics 2, 415 (2008).

[28] T. Kimura, T. Goto, H. Shintani, K. Ishizaka, T. Arima, and Y. Tokura, Magnetic control of ferroelectric polarization, Magnetic control of ferroelectric polarization, Nature (London) 426, 55 (2003).

[29] J. Wang, J. B. Neaton, H. Zheng, V. Nagarajan, S. B. Ogale, B. Liu, D. Viehland, V. Vaithyanathan, D. G. Schlom, U. V. Waghmare, N. A. Spaldin, K. M. Rabe, M. Wuttig, and R. Ramesh, Epitaxial $\mathrm{BiFeO}_{3}$ multiferroic thin film heterostructures, Science 299, 1719 (2003).

[30] Th. Lottermoser, Th. Lonkai, U. Amann, D. Hohlwein, J. Ihringer, and M. Fiebig, Magnetic phase control by an electric field, Nature (London) 430, 541 (2004).

[31] C. H. Ahn, S. Gariglio, P. Paruch, T. Tybell, L. Antognazza, and J.-M. Triscone, Electrostatic modulation of superconductivity in ultrathin $\mathrm{GdBa}_{2} \mathrm{Cu}_{3} \mathrm{O}_{7-x}$ films, Science 284, 1152 (1999).

[32] H. Yamada, M. Kawasaki, Y. Ogawa, and Y. Tokura, Perovskite oxide tricolor superlattices with artificially broken inversion symmetry by interface effects, Appl. Phys. Lett. 81, 4793 (2002). 